\title{
The role of chemokines in inflammatory joint disease
}

\author{
Steven L. Kunkel, Nickolas Lukacs, Tsuyoshi Kasama, ${ }^{\dagger}$ Robert M. Strieter ${ }^{*}$ \\ Departments of Pathology and *Internal Medicine, The University of Michigan Medical School, Ann Arbor, and \\ 'The First Department of Internal Medicine*, Showa University of School of Medicine, Tokyo, Japan
}

\begin{abstract}
Rheumatoid arthritis (RA) is an autoimmune disease characterized by the elicitation and activation of a number of leukocyte populations within both the synovial space and joint tissue. The recruited leukocytes subsequently play an instrumental role in synovial cell proliferation, pannus formation, and bone erosion. Although it is known that leukocytes are important participants in the evolving joint pathology, the mechanism responsible for the succeseful elicitation of cells to the joint is not clear. A number of studies have identified an association of specific cytokines, including chemokines, with active arthritis, but longitudinal analyses of cytokine expression and the causal role of these mediators have not been defined. Animal models and cell culture systems have proved useful in identifying the expression of various cytokines during the maintenance of chronic joint inflammation. In addition, animal models have provided important information regarding the kinetic production and contribution of specific mediators to the development of experimental arthritis. These studies provide insight into the potential mechanisms for leukocyte involvement in inflammatory joint disease. J. Leukoc. Biol. 58: 6-12; 1996.
\end{abstract}

Key Words: monocyte chemotactic protein $1 \cdot$ macrophage inflammatory protein $1 \alpha \cdot c y t o k i n e s \cdot r h e u m a t o i d ~ a r t h r i t i s \cdot l e u k o-$ cytes

\section{INTRODUCTION}

Rheumatoid arthritis (RA) is one of the most common inflammatory joint diseases and has a worldwide distribution. In spite of a large research effort, the pathogenesis of this disease is not clearly understood. RA is often accompanied by significant joint pain and injury caused by active inflammation, leading to synovial cell proliferation, pannus formation, and bone erosion. Clinical management of RA can frequently be difficult, requiring long-term use of potent and often immunosuppressive therapies. These treatment strategies may reflect, in part, the limited understanding of the mechanisms that orchestrate and maintain the joint inflammation. The progression of RA is characterized by the presence of inflammatory cells in both the granuloma-like pannus and the joint fluid, followed by tissue destruction and loss of physical mobility and dexterity. Clinically, it is becoming increasingly clear that specific and efficacious therapeutic interventions must be directed at the active inflammatory cell stage of RA, as end-stage bone erosion and joint fusion is minimally responsive to treatment.

Both recent and historical reports have implicated several mediators in the pathogenesis of RA, including autoantibodies against immunoglobulin $\mathbf{G}$ (rheumatoid factor) and cartilage collagen, immune complexes, complement activation products, oxygen radicals, lysosomal proteases, and several cytokines. Although the proliferation of synovial cells and infiltration of mononuclear leukocytes are fundamental events in the formation of the pannus, it is difficult to examine longitudinally the initiation and maintenance of this pathological cascade in the development of human RA. Therefore, it is necessary to establish and characterize experimental animal models in order to understand the cellular and molecular events that contribute to the pathogenesis of RA. In particular, type II collagen-induced arthritis (CIA) in the mouse has proved to be a useful model of RA, as it has many of the cell-mediated and humoral immunity characteristics found in human RA $[1,2]$. The pathogenesis of CIA is dependent on the host's response to type II collagen challenge and the subsequent generation of antibodies that recognize collagen-rich joint tissue. The chronic activities initiated by immune complexes trigger a variety of cellmediated and humoral events. Recent studies have demonstrated that several cytokines appear to direct cellto-cell communication during the progression of CIA. These cytokines include but are not limited to interleukin-l (IL-1) $[3,4]$, tumor necrosis factor $\alpha$ (TNF- $\alpha)[5,6]$, transforming growth factor $\beta$ (TGF- $\beta$ ) [7], IL-6 [8], and interferon- $\gamma$ (IFN- $\gamma$ ) $[9,10]$. There is little doubt that cytokine-dependent orchestration of immune events is crucial to the pathogenesis of human RA, yet the mechanisms that dictate this pathology have remained unclear. The application of cellular and molecular techniques to both appropriate animal

\footnotetext{
Abbreviations: CIA, collagen-induced arthritis; ELISA, enzyme-linked immunosorbent assay; ENA-78, epithelial neutrophil-activating protein 78; IFN- $\gamma$, interferon- $\gamma$; IL-l, interleukin-l; LPS, lipopolysaccharide; MCP-1, monocyte chemotactic protein 1; MIP-1 $\alpha$, macrophage inflammatory protein $1 \alpha ; R A$, theumatoid arthritis; RANTES, regulated on activation, normal $T$ expressed and secreted; RT-PCR, reverse transcriptase-polymerase chain reaction; TGF- $\beta$, transforming growth factor $\beta$; TNF- $\alpha$, tumor necrosis factor $\alpha$.

Reprint requests: Steven L. Kunkel, Department of Puthology, 1301 Catherine Rd/Box 0602, The University of Michigan Medical School, Ann Arbor, MI 48109-0602.

Received July 26, 1995; accepted August 11, 1995.
} 
models of arthritis and human specimens will prove to be important in addressing these complex issues and provide insight into the development of novel, efficacious therapies.

\section{CHEMOKINE SUPERGENE FAMILIES}

Independent of the etiology, the initiation and maintenance of joint inflammation are due to a dynamic interaction between the inciting stimulus, leukocytes, inflammatory mediators, and resident tissue cells. In the context of chronic joint inflammation, this interaction is often characterized by enhanced permeability, pannus formation and associated mononuclear cell infiltration, and the elicitation of polymorphonuclear leukocytes (PMNs) into the joint fluid. One of the persisting and enigmatic issues of leukocyte chemotaxis is the mechanism(s) that successfully delivers the appropriate leukocyte subtype to a specific area of inflammation. A more complete understanding of the mechanisms of leukocyte chemotaxis have been advanced by studies identifying two supergene families of chemotactic cytokines or chemokines. In their monomeric form, these chemokines are all less than 10,000 daltons and are characterized as basic heparin-binding proteins. These families are structurally identified according to the location of two of four highly conserved cysteine amino acid residues. In one family, the first two amino-terminal cysteines are separated by an additional nonconserved amino acid in a strict C-X-C motif, and the other family possesses a conserved C-C motif near the amino terminus. These chemotactic cytokines are important in inflammation via their specificity for the movement of certain leukocyte populations. In general terms, the C-X-C chemokines possess chemotactic activity for neutrophils, and C-C chemokines possess activity for mononuclear leukocytes (monocytes and lymphocytes). Interestingly, the C-X-C chemokine family are all clustered on human chromosome 4 and demonstrate between 20 and $55 \%$ homology at the amino acid level.

Over the past decade, 12 different human members of the C-X-C supergene family have been discovered with varying activity for neutrophil activation/chemotaxis. They include PF4, amino-terminal truncated forms of platelet basic protein (CTAP-III, beta-thromboglobulin, and NAP-2), GROalpha, macrophage inflammatory protein $2 \alpha$ (MIP-2 $\alpha)$ (GR0-beta), MIP-2 $\beta$ (GR0-gamma), IP-10, MIG, epithelial neutrophil-activating protein 78 (ENA-78), GCP-2, and IL8. The most studied C-X-C chemokine is human IL-8. This chemokine is the prototypic chemotactic member of the C-X-C supergene family and has been found to be produced by a variety of cells. The C-C supergene family comprises a number of structurally related proteins, including MIP$1 \alpha$, MIP-1 $\beta$, macrophage chemotactic protein 1 (MCP-1), MCP-2, MCP-3, MCP-4, RANTES, eotaxin, and I-309. The genes that encode this supergene family reside on human chromosome 17. MCP-1 is one of the most studied of the C-C chemokine family members and has been identified as a chemotactic factor for both monocytes and lymphocytes. Interestingly, it has been demonstrated that MCP-1, MIP-
$1 \alpha$, MIP-1 $\beta$, and RANTES are differentially chemotactic for lymphocyte subsets [11-14]. Whereas a number of studies have centered around the in vitro expression of $C-C$ and $\mathrm{C}$-X-C chemokines from various cellular sources, investigations demonstrating the contribution of these chemokines to the evolution of inflammation in animal models or their role in the pathogenesis of human diseases have not been fully defined.

\section{THE ROLE OF CHEMOKINES IN JOINT DISEASE}

Historically, research directed at understanding the arthritic process has identified the mononuclear phagocyte and lymphocyte as important cellular participants during pannus formation. However, the molecular signals or specific cytokines involved in the elicitation of these cells have remained an enigma. Our current understanding of inflammation suggests that the recruitment of leukocytes from the vascular compartment into a localized area of inflammation is dependent on the initiation and continued maintenance of a chemotactic response. This response is probably dependent on a cascade of events, which must occur with fidelity for the leukocytes to arrive successfully at a site of inflammation. Although many of the steps involved in leukocyte elicitation have been identified, this recruitment process in human joint disease is not completely understood.

There is little doubt that blood-borne monocytes are important effector cells during the evolution of chronic joint disease. The elicitation and activation of these cells during inflammation is a key process, as the monocyte/macrophage can participate in tissue injury via processing and presenting autoantigen(s) and releasing a number of inflammatory mediators, including arachidonic acid and reactive oxygen metabolites, proteolytic enzymes, and a variety of cytokines. The latter protein mediators of inflammation serve as important intercellular signals that orchestrate the initiation and maintenance of arthritis. Recent studies have focused on the mechanism(s) of cytokine/chemokine involvement in the infiltration of leukocytes to synovial tissue in human rheumatoid arthritis $[15,16]$. These studies have identified both MCP-1 and MIP-1 $\alpha$ as likely C-C chemokine candidates responsible for the recruitment of mononuclear cells into the inflamed synovium [15, 16]. Antigenic MCP-1 and MIP-l $\alpha$ were found to be significantly elevated in both synovial fluid and serum of patients with rheumatoid arthritis. Interestingly, patients with osteoarthritis had significantly less MCP-1 and MIP-1 $\alpha$ in both the synovial fluid and serum compared with patients with rheumatoid arthritis. Elevated chemokine levels in the serum were not due to the presence of rheumatoid factor, as there was no statistical correlation between levels of chemokines and rheumatoid factor. Immunolocalization showed that both synovial tissue fibroblasts and macrophages constitutively expressed MCP-1 and MIP-1 $\alpha$. Both chemokines were expressed by cells of the macrophage-rich synovial lining layer from patients with rheumatoid arthritis, whereas normal synovial 
tissue displayed little expression. Macrophages within the subsynovial lining of patients with rheumatoid arthritis were also highly immunopositive for both MCP-1 and MIP$1 \alpha$. Additional studies demonstrated that cultured synovial fibroblasts constitutively expressed MCP-1, which was markedly enhanced by the addition of IL-1, IL-4, or IFN- $\gamma$ [17].

MCP-1 and MIP-1 $\alpha$ are not the only C-C chemokines generated by cells that constitute joint tissue, as synovial fibroblasts isolated from patients with rheumatoid arthritis were found to express steady-state mRNA for the C-C chemokine RANTES and MIP- $1 \beta$. The latter C-C chemokine has been found in association with synovial fibroblasts and macrophages [18]. RANTES mRNA could be detected by in situ hybridization of synovial lining cells in patients with RA; however, in unstimulated cultured synovial fibroblasts RANTES mRNA was undetectable. The addition of either IL-1 or TNF induced a time- and concentrationdependent RANTES expression pattern, which was superinduced upon the addition of cycloheximide. Interestingly, IL-4 down-regulated and IFN- $\gamma$ enhanced TNF- or IL1-stimulated RANTES mRNA expression. Although these studies did not fully assess the fate of RANTES protein or bioactivity, they do lend support to the potential involvement of this $\mathrm{C}-\mathrm{C}$ chemokine in joint inflammation.

The expression of C-X-C chemokines in human rheumatoid arthritis has been explored [15, 16, 19-21]. Chemokines of the C-X-C family were identified as potentially important mediators in the pathogenesis of human rheumatoid arthritis. Synovial fluid recovered from patients with rheumatoid arthritis, but not osteoarthritis, was found to have potent neutrophil chemotactic activity. To determine whether the chemotactic activity was attributable to specific C-X-C chemokines, synovial fluid was preincubated with neutralizing antibody to either IL-8 or ENA-78. This neutralization study reduced the neutrophil chemotactic activity by approximately $57 \%$ and $42 \%$, respectively. Interestingly, the addition of antibodies directed against ENA-78 plus antibodies directed against IL-8 further reduced the chemotactic activity by $70 \%$, suggesting an additive effect of the chemokines on the biologic activity. Subsequent studies showed that the synovial fluid contained significant amounts of antigenic IL-8 and ENA-78, with levels reaching $220 \mathrm{ng} / \mathrm{ml}$. The presence of C-X-C chemokines in the synovial joint fluid was significantly correlated with infiltrating neutrophils in the synovial fluid. Immunolocalization studies assessing the antigenic distribution pattern of IL-8 and ENA-78 demonstrated that both synovial fibroblasts and macrophages expressed these chemokines. The synovial macrophage was the prominent cellular source of antigenic IL-8 and ENA-78. Interestingly, in vitro cultures of isolated unstimulated synovial fibroblasts did not express steady-state levels of IL-8 or ENA-78 mRNA; however, addition of $1 \mathrm{nM}$ of either TNF or IL-1 $\beta$ resulted in the rapid generation of protein and steady-state mRNA levels for these two chemokines. On the contrary, isolated synovial tissue macrophages from patients with rheumatoid arthritis were found to express con- stitutively steady-state levels of IL-8 and ENA-78, which were not further increased upon the addition of TNF or IL-1 $\beta$. Additional investigations demonstrated that steadystate levels of GRO-alpha and GRO-beta, related C-X-C supergene family members, could be detected by synovial fibroblasts isolated from patients with arthritis $[19,20]$. However, these investigations relied on mRNA levels and not on a quantitative assessment of expressed protein. These studies suggested that the synovial fibroblast is dependent on the presence of endogenous mediators that ensure their participation as effector cells during leukocyte recruitment, while activated synovial macrophages appear to be a constant cellular source of specific chemokines during the pathogenesis of arthritis.

\section{CHEMOKINE EXPRESSION VIA CYTOKINE NETWORKS}

Investigations such as those described above support the hypothesis that cytokine networks are operative during the initiation and maintenance of an inflammatory response. The evolution of a cytokine cascade is dependent on the expression of early response cytokines, such as IL-1 or TNF, by tissue macrophages or elicited monocytes/macrophages that may affect surrounding resident tissue cells. This interaction activates the resident cell populations and initiates a number of changes, such as the production of additional cytokines, needed to perpetuate the inflammatory response. Therefore, synovial fibroblast activation in vivo is probably dependent on a cytokine network for the sustained production of chemokines. This scenario is a key aspect of the continued elicitation of leukocytes to the inflamed joint and the maintenance of arthritis. An important concept that has emerged from multiple studies is that resident stromal and parenchymal cells can act as effector cells during the recruitment phase of an immune response. Thus, the nonimmune stromal and parenchymal resident cells that constitute various tissues can no longer be viewed as passive "bystander" cells but must be included as an integral part of the host's immune response. In addition to synovial cells, lung fibroblasts, smooth muscle cells, epithelial cells, and endothelial cells have been identified as important cytokine-secreting cells. A scenario for cytokine networking and the production of chemokines has been addressed in studies in which conditioned media from lipopolysaccharide (LPS)-stimulated tissue macrophages were found to serve as a potent stimulus for the expression of both MCP-1 and IL-8 by human epithelial cells and fibroblasts [22]. The effect of macrophage-conditioned media in these investigations appeared to be mediated, in part, by the paracrine activity of IL-1 and TNF, as chemokine production from stromal cells and parenchymal epithelial cells was not induced by LPS alone and was significantly reduced by neutralizing antibodies to IL-1 $\beta$ and TNF.

A variety of cytokines that are probably involved in initiating inflammation have been studied extensively in the context of both human rheumatoid arthritis and experimen- 
tal models of this disease. For example, endogenous levels of IL-1 are elevated in mice with CIA and the administration of exogenous IL-l to this arthritis model accelerates disease onset. In addition, IL-2, IFN- $\gamma$, and TNF have all been identified as proinflammatory cytokines and contribute to the immunoregulatory network implicated in the pathogenesis of arthritis. Interestingly, a direct role for TNF in joint disease has been implicated in TNF transgenic animals, as these animals develop a chronic inflammatory joint disease characteristic of rheumatoid arthritis. Exogenous additions of IL- 1 , IFN- $\gamma$, and TNF have all resulted in exacerbation of experimental arthritis, but the mechanism for this heightened response is not known. However, cytokine cascades are probably important mechanisms involved in the movement of mononuclear cells from the peripheral circulation into the developing pannus and neutrophils to the joint fluid during the initiation and maintenance of rheumatoid arthritis. Studies directed at understanding the cells and mediators that initiate and maintain this inflammatory response are critically important, as effective therapeutic strategies must be directed at mediators involved in the cellular phase of these disorders.

\section{ENDOGENOUS MODULATION OF CYTOKINES AND THEIR POTENTIAL TO ALTER JOINT DISEASE}

The ability of the host to establish cytokine networks is an important aspect of the initiation of inflammation. This amplified cascade also allows for a number of specific points of regulation and potential intervention. The importance of a regulated cytokine response during inflammation is underscored by the identification of endogenous cytokine inhibitory proteins [23]. Some of these host-derived inhibitory proteins have been shown to block the biological activity of early response cytokines, such as IL-1 and TNF $[24,25]$, while others have broad suppressive effects on cytokine production [26]. The mechanism of these inhibitors appears to be either interference with the formation of a functional cytokine-cell surface receptor complex, thus negating classic cell signaling events, or blocking of cytokine protein expression. The importance of soluble cytokine receptors in the regulation of inflammation is highlighted by the interesting observation that various poxviruses encode polypeptides with both functional and structural homology to the extracellular sections of the TNF, IL-1, and IFN- $\gamma$ receptors, suggesting that these virus-derived receptor homologues aid the virus in subverting the host's immune response. In addition to the viral expression of soluble cytokine receptors, the cytokine IL-10, originally identified as cytokine synthesis inhibitory factor, has a virus-derived counterpart. A high degree of structural and functional homology exists between IL-10 and a protein (BCRFI) encoded by the Epstein-Barr virus. These homologies underscore the importance of endogenous regulatory factors in modulating the inflammatory response, as the production of these "captured genes" during viral infections probably aid in limiting the normal inflammatory response.

Investigations have demonstrated that endogenous regulators of cytokine production or activity are expressed during human rheumatoid arthritis or during the development of experimental arthritis. Purified synovial macrophages from humans with rheumatoid arthritis have been shown to produce constitutively IL-1 receptor antagonist protein (IRAP), whereas normal blood monocytes exhibit little constitutive IRAP expression. Immunohistochemical localization studies have identified synovial tissue macrophages, as well as synovial lining cells, as important IRAP-expressing cells in the inflamed joint tissue. Additional studies identified significant production of IRAP within the arthritic lesion and also found the infiltrating neutrophils to be a rich cellular source of IRAP. These original studies were expanded to demonstrate that TNF receptors could be localized to the synovial tissue and pannus in patients with rheumatoid arthritis. The potential regulatory influence of IRAP and the soluble TNF receptor on the development of experimental arthritis has been shown in the type II collagen-induced arthritis model. Exogenous IRAP, recombinant sTNFr, or an sTNF:Fc antibody construct have been identified as ameliorating the physical parameters associated with this model of joint inflammation.

The presence and role of IL-10, a potent cytokine synthesis inhibitory factor, has also been assessed in cells from humans with rheumatoid arthritis. In this study, the expression of both mRNA and protein for IL-10 could be demonstrated in rheumatoid arthritis joints. Human IL-10 mRNA could be demonstrated by reverse transcriptase-polymerase chain reaction (RT-PCR) amplification of total RNA isolated directly from synovial tissue. In addition, IL-10 protein was spontaneously produced in synovial membrane cultures. Immunolocalization analysis demonstrated that mononuclear phagocytic cells were the main source of IL10 in the synovial lining layer. The addition of exogenous IL-10 to cultured synoviocytes caused a two- to threefold decrease in the levels of both IL-1 and TNF, while IL-6 production was not affected. However, the addition of neutralizing anti-IL-10 antibodies to the cultured synoviocytes caused an increase in the expression of the same proinflammatory mediators.

\section{CHEMOKINE EXPRESSION DURING THE EVOLUTION OF EXPERIMENTAL JOINT INFLAMMATION}

While the assessment of clinical specimens is important in beginning to understand the contribution of mediators to the inflammatory response and the developing disease process, this approach is often difficult for either ethical, availability, or logistic reasons. In addition, observations related to human samples often lack a causal relationship between mediator and disease, providing only a one-time "snapshot" of the evolving pathology. Therefore, experimental animal models are important for understanding certain mechanisms involved in the pathogenesis of disease. This is espe- 
TABLE 1. Kinetic Analysis of MCP-1, MIP-1 $\alpha$, and IL-10 Levels in Aqueous Extracts of Joint Tissue from Mice with CIA $^{a}$

\begin{tabular}{lccc}
\hline \hline & \multicolumn{3}{c}{ Cytokine (pq/ml) } \\
\cline { 2 - 4 } Daye after challenge & MIP-1 $\alpha$ & MCP-1 & IL-10 \\
\hline 32 & 110 & 100 & 250 \\
36 & 150 & 300 & 280 \\
40 & 200 & 600 & 300 \\
44 & 270 & 780 & 540 \\
48 & 250 & 600 & 590 \\
52 & 210 & 610 & 595 \\
56 & 200 & 600 & 610 \\
60 & 190 & 600 & 590 \\
\hline
\end{tabular}

'Samples were taken every four days from time of initial collagen challenge. There was no significant difference between control and CIA animals until day 36 .

cially true of chronic diseases, in which longitudinal studies in humans are extremely difficult to perform. Our previous investigations demonstrate that specific chemokines are probably important in the pathophysiology of human rheumatoid arthritis $[15,16]$; however, their mechanism of regulation, sequential expression patterns, and contribution to the pathogenesis of arthritis remain to be fully elucidated. To address these important questions related to chemokine involvement during the initiation and maintenance of joint inflammation, we have utilized the established, reproducible, and well-described model of type II collagen-induced arthritis (CIA) in mice (DBA/1J).

The recruitment of neutrophils and mononuclear cells into the inflamed joint is a crucial event in the development of arthritis. We initially assessed the expression of antigenic MIP-1 $\alpha$ and MIP-2 in sections of the arthritic joint. Immunohistology identified the expression of both MIP- $1 \alpha$ and MIP-2 by infiltrating macrophage-like cells in the joint at 68 days after primary immunization, whereas control antibodies demonstrated no nonspecific staining. MIP-l $\alpha$ was also found to be produced by chondrocytes and fibroblast-like cells in the joint tissue. Next, we longitudinally assessed the expression pattern for both C-C and C-X-C chemokines in aqueous joint tissue extracts (Table 1). Mice with or without immunization with type II collagen were sacrificed every 4 days and the four footpads of each mouse, minus the skin, were homogenized and chemokines assessed by ELISA. This expression pattern was kinetically associated with the infiltration of leukocytes and the physical signs of the incidence and index of arthritis. By day 36 after immunization with type II collagen, elevated levels of MIP- $1 \alpha$ and MCP-1 (C-C chemokines) and MIP-2 (a C-X-C chemokine) could be observed. During the later time points of this inflammatory response significant elevation of all of the chemokines can be found, which correlates with significant elicitation of leukocytes into the inflamed joint. The expression pattern for antigenic MIP-1 $\alpha$ and MIP-2 paralleled a similar timedependent expression of steady-state levels of MIP-l $\alpha$ and MIP-2 mRNA by RT-PCR. Steady-state levels of MIP-2 mRNA appeared by approximately day 12; both MIP-2 and MIP-1 $\alpha$ mRNAs peaked at days 36 to 44 .

\section{PASSIVE IMMUNIZATION WITH ANTI-MIP-1 $\alpha$ OR ANTI-MIP-2 ANTIBODIES AMELIORATES JOINT INFLAMMATION}

The preceding investigations demonstrated that steadystate mRNA and protein levels of specific chemokines are temporally associated with the recruitment of leukocytes into inflamed joints and their expression pattern correlates with physical signs of the disease. In order to determine the potential contribution of specific chemokines to the developing joint disease, mice were passively immunized with antibodies directed against either MIP-1 $\alpha$, MIP-2, or control antibodies. In this study we utilized $F\left(a^{\prime}\right)_{2}$ fragments, as immune complexes between various antibodies and collagen, or other factors, may alter the normal development of the CIA response. Mice were treated intraperitoneally with $1 \mathrm{mg}$ of neutralizing $\mathrm{F}\left(\mathrm{ab}^{\prime}\right)_{2}$ every other day from days 24 to 32 and joints were individually scored in a "blinded" fashion on a scale of 0 to 3 , maximum score of 12 per mouse, on the amount of erythema, swelling, or joint rigidity (arthritis index). Mice were also assessed for the percent incidence of arthritis, as determined by the percentage of affected joints. As shown in Table 2, passive immunization with antibodies to MIP-1 $\alpha$ or MIP-2 resulted in a significant reduction of the observed arthritis, suggesting that targeting individual chemokines may prove to be a beneficial therapeutic approach to attenuating arthritis.

TABLE 2. Kinetic Analysis of the Effects of Passive Immunization with Anti-MIP-2 or Anti-MIP-1 $\alpha$ on the Arthritis Index and \% Incidence of Arthritis in Mice with Developing $\mathrm{CIA}^{a}$

\begin{tabular}{|c|c|c|c|c|c|c|c|c|}
\hline \multirow[b]{3}{*}{ Days } & \multicolumn{4}{|c|}{ Anti-MIP-2 } & \multicolumn{4}{|c|}{ Anti-MIP-1 $\alpha$} \\
\hline & \multicolumn{2}{|c|}{ Arthritis index } & \multicolumn{2}{|c|}{$\%$ incidence } & \multicolumn{2}{|c|}{ Arthritis index } & \multicolumn{2}{|c|}{$\%$ incidence } \\
\hline & Cont & Anti & Cont & Anti & Cont & Anti & Cont & Anti \\
\hline 32 & 1 & 0 & 25 & 0 & 1 & 0 & 25 & 0 \\
\hline 36 & 2 & .5 & 30 & 30 & 2 & .5 & 30 & 30 \\
\hline 40 & 2.2 & 1 & 50 & 50 & 2.2 & 1 & 50 & 50 \\
\hline 44 & 3.8 & 1.8 & 72 & 50 & 3.8 & 1.7 & 72 & 50 \\
\hline 48 & 4.2 & 3 & 72 & 50 & 4.2 & 1.5 & 72 & 50 \\
\hline 52 & 6 & 3.8 & 72 & 50 & 6 & 2 & 72 & 50 \\
\hline 56 & 7.2 & 3.8 & 100 & 70 & 7.2 & 3 & 100 & 50 \\
\hline 60 & 7.8 & 4.2 & 100 & 74 & 8 & 3.8 & 100 & 74 \\
\hline
\end{tabular}

-Data represent subjective determination of the severity and onset of the joint inflammation. Cont, control; Anti, antibody. 


\section{EXPRESSION AND CONTRIBUTION OF IL-10 DURING JOINT INFLAMMATION}

A number of in vitro and in vivo investigations have identified IL-10 as an important regulatory cytokine during the development of an immune/inflammatory response. Thus, it was of interest to determine the kinetic expression of IL-10 during the development of CIA (Table 1). A significant elevation in levels of IL-10 protein was identified at approximately day 44 after primary immunization with type II collagen. The kinetic pattern of IL-10 protein, as determined by a specific ELISA for murine IL-10, was substantiated by RT-PCR for the expression of steady-state IL-10 mRNA. This time frame for IL-10 protein and mRNA was coincident with the development of arthritis. Interestingly, IL-10 was found associated with infiltrating macrophagelike cells, chondrocytes, and fibroblast-like cells by immunohistology. Fibroblast-like cells from the inflamed joint expressed significant levels of IL-10 antigen, which was further substantiated by immunostaining of cultured synovial fibroblast-like cells.

\section{PASSIVE IMMUNIZATION WITH ANTI-IL-10 AUGMENTS JOINT CHEMOKINE EXPRESSION}

Previous studies by our group and others have identified an augmentation in the production of specific cytokines after in vivo administration of anti-IL-10 antibodies. In addition, the data above suggest that anti-IL-10 antibody treatment increased disease severity and augmented leukocyte elicitation. To begin assessing the mechanism(s) responsible for the increased inflammatory response, we next determined the effect of anti-IL-10 antibody therapy on the levels of MIP-1 $\alpha$ and MIP-2. Again, F $\left(a^{\prime}\right)_{2}$ fragments of IL-10 antibody were administered to mice every other day from days 24 to 32 after primary immunization with type II collagen. Aqueous joint tissue extracts were then determined at day 48. Both MIP-1 $\alpha$ and MIP-2 levels were significantly elevated in the anti-IL-10 antibody-treated animals. These data parallel the histologic observations, which demonstrated an increase in leukocyte infiltrates in the inflamed joint, a process due, in part, to the overexpression of chemokines.

Taken together, the studies described here demonstrate that specific chemokines are important to the initiation and maintenance of arthritis, and endogenous IL-10 plays a crucial role in regulating the expression of proinflammatory cytokines, including specific chemokines.

\section{ACKNOWLEDGMENTS}

These studies were supported in part by National Institutes of Health grants 1POHL46487, HL35276, HL31963, HL31237, and HL50057.

\section{REFERENCES}

1. Stuart, J.M., Townes, A.S., Kang, A.H. (1984) Collagen autoimmune arthritis. Annu. Rev. Immunol. 2, 199-218.

2. Holmdahl, R., Andersson, M., Goldschmidt, T.J., Gustafsson, K., Jansson, L., Mo, J.A. (1990) Type II collagen autoimmunity in animals and provocations leading to arthritis. Immunol. Rev. 118, 193-232.

3. Hom, J.T., Cole, H., Estridge, T., Gliszczynski, V.L. (1992) Interleukin-1 enhances the development of type II collagen-induced arthritis only in susceptible and not in resistant mice. Clin. Immunol. Immunopathol. 62, 56-65.

4. Hom, J.T., Bendele, A.M., Carlson, D.G. (1988) In vivo administration with IL-1 accelerates the development of collagen-induced arthritis in mice. J. Immunol. 141, 834-841.

5. Piguet, P.F., Grau, G.E., Vesin, C., Loetscher, H., Gentz, R., Lesslauer, W. (1992) Evolution of collagen arthritis in mice is arrested by treatment with anti-tumour necrosis factor (TNF) antibody or a recombinant soluble TNF receptor. Immunology 77, 510-514.

6. Williams, R.O., Feldmann, M., Maini, R.N. (1992) Anti-tumor necrosis factor amelionates joint disease in murine collagen-induced arthritis. Proc. Natl. Acad. Sci. USA 89, 9784-9788.

7. Thorbecke, G.J., Shah, R., Leu, C.H., Kuruvilla, A.P., Hardis, W. (1992) Involvement of endogenous tumor necrosis factor alpha and transformin growth factor beta during induction of collagen type II arthritis in mice. Proc. Natl. Acad. Sci. USA 89, 7375-7379.

8. Takai, Y., Seki, N., Senoh, H. (1989) Enhanced production of interleukin-6 in mice with type II collagen-induced arthritis. Arthritis Rheum. 32, $594-600$.

9. Cooper, S.M., Sriram, S., Ranges, G.E. (1988) Suppression of murine collagen-induced arthritis with monoclonal anti-la antibodies and augmentation with IFN-gamma. J. Immunol. 141, 1958-1962.

10. Mauritz, N.J., Holmdahlm, R., Jonssonm, R., Van der Meidem, P.H., Scheyniusm, A., Klareskog, L. (1988) Treatment with gamma-interferon triggers the onset of collagen arthritis in mice. Arthritis Rhewm. 31, 1297-1304.

11. Oppenheim, JJ., Zachariae, O.C., Mukaida, N., Matsushima, K. (1991) Properties of the novel proinflammatory supergene "intercrine" cytokine family. Annu. Rev. Immunol. 9, 617-648.

12. Larsen, C.G., Anderson, A.O., Appella, E., Oppenheim, JJ., Matsushima, K. (1989) Identity of the chemotactic cytokine for T-lymphocytes with neutrophil activating protein (NAP-1): a candidate interleukin-8. Science 243, $1464-1466$

13. Schall, T.J., Bascon, K.J., Toy, K.J., Goeddel, D.V. (1990) Selective attraction of monocytes and $\mathrm{T}$ lymphocytes of the memory phenotype by cytokine RANTES. Nature 347, 669-672.

14. Tanaka, Y., Adams, D.H., Hubscher, S., Hirano, H., Siebenlist, U., Shaw, S (1993) T-cell adhesion induced by proteoglycan-immobilized cytokine MIP-1 beta. Nature 361, 79-82.

15. Koch, A.E., Kunkel, S.L., Harlow, B., Johnson, H.L., Evanoff, H.L., Haines, G.K., Burdick, M.D., Strieter, R.M. (1992) Enhanced production of monocyte chemoattractant protein-1 in theumatoid arthritis. J. Clin. Invest. 90 , $772-779$.

16. Koch, A.E., Kunkel, S.L., Harlow, L.A., Mazarakis, D.D., Haines, G.K., Burdick, M.D., Pope, R.M., Strieter, R.M. (1994) Macrophage inflammatory protein-1. A novel chemotactic cytokine for macrophages in theumatoid arthritis. J. Clin. Invest. 93, 921-928.

17. Seitz, M., Loetscher, P., Dewald, B., Towbin, H., Ceska, M., Baggiolini, M. (1994) Production of IRAP, inflammatory chemotactic proteins, and prostaglandin $E$ by theumatoid and osteoarthritic synoviocytes - regulation by IFN-gamma and IL-4. J. Immunol. 152, 2060-2065.

18. Kasama, T., Strieter, R.M., Lukacs, N.W., Lincoln, P.M., Burdick, M.D., Kunkel, S.L. (1995) Interleukin-10 expression and chemokine regulation during the evolution of murine type II collagen-induced arthritis. J. Clin. Invest. 95, 2868-2876.

19. Hogan, M., Sherry, B., Ritchlin, C., Fabre, M., Winchester, R., Cerami, A., Bucala, R. (1994) Differential expression of the small inducible cytokine GRO alpha and GRO beta by synovial fibroblasts in chronic arthritis: possible role in growth regulation. Cytokine 6, 61-69.

20. Unemori, E.N., Amento, E.P., Bauer, E.A., Horuk, R. (1993) Melanoma growth-stimulatory activity/GRO decreases collagen expression by human fibroblasts. J. Biol. Chem. 268, 1338-1342.

21. Beaulieu, A.D., McColl, S.R. (1994) Differential expression of two major cytokines produced by neutrophils, IL-8 and the interleukin-1 receptor antagonist, in neutrophils isolated from the synovial fluid and peripheral blood of patients with theumatoid arthritis. Arthritis Rheum. 37, 855-859.

22. Rolfe, M.W., Kunkel, S.L., Standiford, T.J., Orringer, M.B., Phan, S.H., Evanoff, H.L., Burdick, M.D.,Strieter, R.M. (1992) Expression and regulation of human pulmonary fibroblast-derived monocyte chemotactic peptide-1.Am. J. Physiol. 263, 536-545.

23. de Waal Malefyt, R., Abrams, J., Bennett, B., Figdor, C.G., DeVries, J.E. (1991) Interleukin-10 inhibits cytokine synthesis by human monocytes: an autoregulatory role of IL-10 produced by monocytes. J. Exp. Med. 174, 1209-1220. 
24. Larrick, J.W. (1989) Native interleukin-l inhibitors. Immunol. Today 10,61-66. 25. Mohler, K.M., Torrance, D.S., Smith, C.A., Goodwin, R.G., Stremler, K.E. Fung, V.P., Madani, H., Widmer, M.B. (1993) Soluble TNF receptors are effective therapeutic agents in lethal endotoxemia and function simultaneously as both TNF carriers and TNF antagonists. J. Immunol. 151, 1548-1561.
26. Vieira, P., Waal-Malefyt, R.D., Dang, M.N., Johnson, K.E., Kastelein, R., Fiorentino, D.F., DeVries, J.E., Roncarolo, M.G., Mosmann, T.R., Moore, K.W. (1991) Isolation and expression of human cytokine synthesis inhibitory factor cDNA clones: homology to Epstein-Barr virus open reading frame BCRFI. Proc. Natl. Acad. Sci. USA 88, 1172-1176. 